\title{
Truncated Euler-Maruyama method for classical and time-changed non-autonomous stochastic differential equations
}

\author{
Wei Liu ${ }^{\mathrm{a}}$, Xuerong Mao ${ }^{\mathrm{b}}$, Jingwen Tang ${ }^{\mathrm{a}, *}$, Yue $\mathrm{Wu}^{\mathrm{c}, \mathrm{d}}$ \\ ${ }^{a}$ Department of Mathematics, Shanghai Normal University, Shanghai, 200234, China \\ ${ }^{b}$ Department of Mathematics and Statistics, University of Strathclyde, Glasgow, G1 1 XH, \\ $U K$ \\ ${ }^{c}$ School of Engineering, University of Edinburgh, Edinburgh, EH9 3JW, UK \\ ${ }^{d}$ Mathematical Institute, University of Oxford, Oxford, OX2 6GG, UK
}

\begin{abstract}
The truncated Euler-Maruyama (EM) method is proposed to approximate a class of non-autonomous stochastic differential equations (SDEs) with the Hölder continuity in the temporal variable and the super-linear growth in the state variable. The strong convergence with the convergence rate is proved. Moreover, the strong convergence of the truncated EM method for a class of highly non-linear time-changed SDEs is studied.

Keywords: Truncated Euler-Maruyama method, non-autonomous stochastic differential equations, strong convergence, super-linear coefficients, time-changed stochastic differential equations.
\end{abstract}

2010 MSC: 65C30, 65L20, 60H10

\section{Introduction}

2 Stochastic differential equations (SDEs) have broad applications in many areas such as finance, physics, chemistry and biology [1, 29]. However, most SDEs do not have the explicit expressions of the true solutions. Therefore, 5 the numerical methods and the rigorous numerical analyses of those methods 6 become extremely important $[17,27]$.

\footnotetext{
* Corresponding author

Email address: j.w.tang@foxmail.com (Jingwen Tang )
}

Preprint submitted to a journal

February 11, 2020 
In this paper, we investigate the numerical approximation to the solutions of a class of non-autonomous stochastic differential equations of the Itô type

$$
\left\{\begin{array}{l}
\mathrm{d} x(t)=\mu(t, x(t)) \mathrm{d} t+\sum_{r=1}^{m} \sigma^{r}(t, x(t)) \mathrm{d} W^{r}(t), \quad t \in\left[t_{0}, T\right], \\
x\left(t_{0}\right)=x_{0},
\end{array}\right.
$$

where the coefficients obey the Hölder continuity in the temporal variable and the super-linear growth condition in the state variable. The detailed mathematical descriptions can be found in Section 2.

For non-autonomous SDEs with the Hölder continuous temporal variable in the coefficients, the randomized techniques are used to construct the Euler type method [30] and the Milstein type method [19]. However, most papers that investigate non-autonomous SDEs only consider the global Lipschitz condition for the state variable. Thus, one aim of this paper is to study the non-autonomous SDEs whose coefficients may grow super-linearly in the state variable, known as highly non-linear SDEs.

The classic Euler-Maruyama (EM) method has been proved divergent for highly non-linear SDEs [11]. While bearing in mind the idea that explicit methods have their advantages in simple algorithm structure and relatively lower computational cost in the simulations of a large number of sample paths [9], the tamed Euler method [12] and the truncated EM method [24] are developed 22 to approximate the solutions of highly non-linear SDEs. Some other interest${ }_{23}$ ing works on explicit methods for highly non-linear SDEs are, for example, ${ }_{24}[3,7,8,10,13,20,31,33,35,36,38]$ and the references therein. However, those 25 explicit methods proposed to tackle the super-linearity in the state variable do 26 not take the non-autonomous SDEs into consideration.

When both the Hölder continuity in the temporal variable and the superlinearity in the state variable appear together in one SDE, few works have been done on the numerical approximation to its solution. To fill up this gap, we investigate the truncated EM method for this type of SDEs in this paper.

The time-changed SDEs, where the time variable $t$ is replaced by some stochastic process $E(t)$ (see Section 4 for the details), have attracted lots of 
attentions in recent years $[5,22,25,28,32,34,37]$. Due to the change of the time, the solution to the time-changed SDE is understood as a subdiffusion process, which could be used to describe diffusion phenomena that move slower than the Brownian motion [2, 26]. Numerical approximations to such type of SDEs are also important, as the explicit forms of the true solutions are rarely obtained. Only recently, authors in [16] studied the classical EM method for a class of time-changed SDEs, both of whose drift and diffusion coefficients satisfy the global Lipschitz condition. To our best knowledge, [16] is the first paper to investigate the numerical approximation to time-changed SDEs by directly discretising the equations. More recently, the semi-implicit EM method was proposed in [4] to approximate some time-changed SDEs with the global Lipschitz condition on the drift coefficient being replaced by the one-sided Lipschitz condition. Both of those two works used the duality principle proposed in [18], which, briefly speaking, relates the time-changed SDEs to certain kind of SDEs (see Section 4 for more details). In [14], the authors investigated the classical EM method for a larger class of time-changed SDEs without the application of the duality principle, though the drift and diffusion coefficients still satisfy the global Lipschitz condition. All the three works $[4,14,16]$ investigated either the $L^{1}$ or $L^{2}$ convergence.

In this paper, the truncated EM method is used to approximate a class of time-changed SDEs of the form

$$
d y(t)=\mu(E(t), y(t)) d E(t)+\sigma(E(t), y(t)) d W(E(t)) .
$$

To our best knowledge, this is the first work devoted to numerical approximations to time-changed SDEs, whose drift and diffusion coefficients are allowed to grow super-linearly. Moreover, we consider the $L^{\bar{q}}$ convergence for any $\bar{q} \geq 2$.

The main contributions of our work are as follows.

- The truncated EM method, which is an explicit method, is proved to be convergent to SDEs with the Hölder continuity in the temporal variable and the super-linearity in the state variable. 
- The convergence rate of $\min \left\{\alpha, \gamma, \frac{1}{2}-\varepsilon\right\}$ is given, where $\alpha$ and $\gamma$ are the Hölder continuity indexes in the drift and diffusion coefficients, and $\varepsilon>0$ could be arbitrarily small.

- The strong convergence of the truncated EM method for a class of timechanged SDEs, whose coefficients can grow super-linearly, is proved.

The paper is constructed as follows. Section 2 briefly introduces the truncated EM method and some useful lemmas. The strong convergence with the rate for classical SDEs is presented and proved in Section 3. The truncated EM method for time-changed SDEs is discussed in 4. Numerical examples are given in Section 5 to demonstrate the theoretical results.

\section{Mathematical preliminaries}

This section is divided into three parts. In Section 2.1, the notations and assumptions are introduced. To keep the paper self-contained, the truncated EM method is briefed in Section 2.2. Some useful lemmas are presented in Section 2.3 .

\subsection{Notations and assumptions}

Throughout this paper, unless otherwise specified, we let $\left(\Omega_{W}, \mathcal{F}^{W}, \mathbb{P}_{W}\right)$ be a complete probability space with a filtration $\left\{\mathcal{F}_{t}^{W}\right\}_{t \in[0, T]}$ satisfying the usual conditions (that is, it is right continuous and increasing while $\mathcal{F}_{0}^{W}$ contains all $\mathbb{P}_{W}$-null sets), and let $\mathbb{E}_{W}$ denote the probability expectation with respect to $\mathbb{P}_{W}$. If $x \in \mathbb{R}^{d}$, then $|x|$ is the Euclidean norm. Let $x^{T}$ denote the transposition of $x$. Moreover, for two real numbers $a$ and $b$, we use $a \vee b=\max (a, b)$ and $a \wedge b=\min (a, b)$.

For $d, m \in \mathbb{N}$, let $W:\left[t_{0}, T\right] \times \Omega_{W} \rightarrow \mathbb{R}^{m}$ be a standard $\left\{\mathcal{F}_{t}^{W}\right\}_{t \in\left[t_{0}, T\right]}$-Wiener process. Moreover, let $x:\left[t_{0}, T\right] \times \Omega_{W} \rightarrow \mathbb{R}^{d}$ be an $\left\{\mathcal{F}_{t}^{W}\right\}_{t \in\left[t_{0}, T\right]}$-adapted stochastic process that is a solution to Itô type stochastic differential equation 


$$
\left\{\begin{array}{l}
\mathrm{d} x(t)=\mu(t, x(t)) \mathrm{d} t+\sum_{r=1}^{m} \sigma^{r}(t, x(t)) \mathrm{d} W^{r}(t), \quad t \in\left[t_{0}, T\right], \\
x\left(t_{0}\right)=x_{0},
\end{array}\right.
$$

${ }_{85}$ where $\mathbb{E}_{W}\left|x_{0}\right|^{p}<\infty$ for any $p>0$, the drift coefficient function $\mu:\left[t_{0}, T\right] \times$

${ }_{86} \mathbb{R}^{d} \rightarrow \mathbb{R}^{d}$ and the diffusion coefficient function $\sigma^{r}:\left[t_{0}, T\right] \times \mathbb{R}^{d} \rightarrow \mathbb{R}^{d}$ for 87 $r \in\{1,2, \cdots, m\}$.

88 We impose the following assumptions on the drift and diffusion coefficients.

Assumption 2.1. Assume that there exist positive constants $\beta$ and $M$ such that

$$
|\mu(t, x)-\mu(t, y)| \vee\left|\sigma^{r}(t, x)-\sigma^{r}(t, y)\right| \leqslant M\left(1+|x|^{\beta}+|y|^{\beta}\right)|x-y|,
$$

s9 for all $t \in\left[t_{0}, T\right]$, any $x, y \in \mathbb{R}^{d}$ and any $r \in\{1,2, \cdots, m\}$.

90 It can be observed from Assumption 2.1 that all $t \in\left[t_{0}, T\right], r \in\{1,2, \cdots, m\}$ $91 \quad$ and $x \in \mathbb{R}^{d}$

$$
|\mu(t, x)| \vee\left|\sigma^{r}(t, x)\right| \leq K\left(1+|x|^{\beta+1}\right),
$$

$92 \quad$ where $K$ depends on $M$ and $\sup _{t_{0} \leq t \leq T}\left(|\mu(t, 0)|+\max _{1 \leq r \leq m}\left|\sigma^{r}(t, 0)\right|\right)$.

Assumption 2.2. Assume that there exists a pair of constants $q>2$ and $L_{1}>0$ such that

$$
(x-y)^{T}(\mu(t, x)-\mu(t, y))+\frac{q-1}{2} \sum_{r=1}^{m}\left|\sigma^{r}(t, x)-\sigma^{r}(t, y)\right|^{2} \leq L_{1}|x-y|^{2},
$$

${ }_{93}$ for all $t \in\left[t_{0}, T\right]$ and any $x, y \in \mathbb{R}^{d}$.

Assumption 2.3. Assume that there exists a pair of constants $p>2$ and $L_{2}>0$ such that

$$
x^{T} \mu(t, x)+\frac{p-1}{2} \sum_{r=1}^{m}\left|\sigma^{r}(t, x)\right|^{2} \leq L_{2}\left(1+|x|^{2}\right),
$$

${ }_{94}$ for all $t \in\left[t_{0}, T\right]$ and any $x \in \mathbb{R}^{d}$.

95 Remark 2.4. It is clear that Assumption 2.3 may be derived from Assumption

962.2 but with more complicated coefficient in front of $\left|\sigma^{r}(t, x)\right|^{2}$. To keep the ${ }_{97}$ notation simple, we state Assumption 2.3 as a new assumption. 
where we set $x /|x|=0$ when $x=0$.

Define the truncated functions by

$$
\mu_{\Delta}(t, x)=\mu\left(t, \pi_{\Delta}(x)\right), \quad \sigma_{\Delta}(t, x)=\sigma\left(t, \pi_{\Delta}(x)\right),
$$

for $x \in \mathbb{R}^{d}$. It is easy to see that for any $t \in\left[t_{0}, T\right]$ and all $x \in \mathbb{R}^{d}$

$$
\left|\mu_{\Delta}(t, x)\right| \vee\left|\sigma_{\Delta}(t, x)\right| \leq f\left(f^{-1}(\kappa(\Delta))\right)=\kappa(\Delta) .
$$


The discrete-time truncated EM numerical solutions $x_{\Delta}\left(t_{k}\right)$, to approximate $x\left(t_{k}\right)$ for $t_{k}=k \Delta+t_{0}$, are formed by setting $x_{\Delta}\left(t_{0}\right)=x_{0}$ and computing

$$
x_{\Delta}\left(t_{k+1}\right)=x_{\Delta}\left(t_{k}\right)+\mu_{\Delta}\left(t_{k}, x_{\Delta}\left(t_{k}\right)\right) \Delta+\sum_{r=1}^{m} \sigma_{\Delta}^{r}\left(t_{k}, x_{\Delta}\left(t_{k}\right)\right) \Delta W_{k}^{r},
$$

for $k=0,1, \cdots, N_{\Delta}$, where $N_{\Delta}$ is the integer part of $T / \Delta$ and we will set $t_{N_{\Delta}+1}=T$ while $\Delta W_{k}^{r}=W^{r}\left(t_{k+1}\right)-W^{r}\left(t_{k}\right)$.

To form the continuous versions of truncated EM numerical schemes, we define

$$
\tau(t)=\sum_{k=0}^{N_{\Delta}} t_{k} I_{\left[t_{k}, t_{k+1}\right)}(t), \quad t \in\left[t_{0}, T\right] .
$$

There are two versions of the continuous-time truncated EM solutions. The first one is defined by

$$
\bar{x}_{\Delta}(t)=\sum_{k=0}^{N_{\Delta}} x_{\Delta}\left(t_{k}\right) I_{\left[t_{k}, t_{k+1}\right)}(t)
$$

which is a simple step process. The other one is defined by

$$
x_{\Delta}(t)=x_{0}+\int_{t_{0}}^{t} \mu_{\Delta}\left(\tau(s), \bar{x}_{\Delta}(s)\right) d s+\sum_{r=1}^{m} \int_{t_{0}}^{t} \sigma_{\Delta}^{r}\left(\tau(s), \bar{x}_{\Delta}(s)\right) \mathrm{d} W^{r}(s),
$$

which is continuous in $t \in\left[t_{0}, T\right]$.

\subsection{Some useful lemmas}

In this subsection, some lemmas that will be essential for the proof of the main result in Section 3 are presented. The proofs of these lemmas are either straightforward or can be found in references. Therefore, to focus our attention on the proof of the main result, those lemmas are stated without proofs.

Lemma 2.6. Let Assumptions 2.1 and 2.3 hold. The SDE (1) has a unique global solution $x(t)$. Moreover,

$$
\sup _{t_{0} \leq t \leq T} \mathbb{E}_{W}|x(t)|^{p}<\infty .
$$

The proof of the above lemma can be found in , for example, Theorem 4.1 in Page 59 of [23]. 
Lemma 2.7. For any $\Delta \in(0,1]$ and any $\bar{p}>0$, we have

$$
\mathbb{E}_{W}\left|x_{\Delta}(t)-\bar{x}_{\Delta}(t)\right|^{\bar{p}} \leq C_{\bar{p}} \Delta^{\frac{\bar{p}}{2}}(\kappa(\Delta))^{\bar{p}}, \quad \forall t \in\left[t_{0}, T\right],
$$

where $C_{\bar{p}}$ is a positive constant dependent only on $\bar{p}$. Consequently

$$
\lim _{\Delta \rightarrow 0} \mathbb{E}_{W}\left|x_{\Delta}(t)-\bar{x}_{\Delta}(t)\right|^{\bar{p}}=0, \quad \forall t \in\left[t_{0}, T\right] .
$$

Lemma 2.8. Let Assumptions 2.1 and 2.3 hold. Then

$$
\sup _{0<\Delta \leq 1} \sup _{t_{0} \leq t \leq T} \mathbb{E}_{W}\left|x_{\Delta}(t)\right|^{p} \leq C,
$$

where $C$ is a positive constant independent of $\Delta$.

From now on, the constants $C, C_{1}, C_{2}, C_{3}, C_{31}$ and $C_{32}$ stand for generic positive constants that are independent of $\Delta$ and their values may change between occurrences.

The proofs of Lemmas 2.7 and 2.8 follow straightforwardly from the proofs of Lemmas 3.1 and 3.2 in [24], by substituting $\mu_{\Delta}\left(t, \bar{x}_{\Delta}(s)\right)$ and $\sigma_{\Delta}\left(t, \bar{x}_{\Delta}(s)\right)$ for $\mu_{\Delta}\left(\bar{x}_{\Delta}(s)\right)$ and $\sigma_{\Delta}\left(\bar{x}_{\Delta}(s)\right)$, respectively.

Remark 2.9. From Lemma 2.8 , it is easily obtained that

$$
\sup _{0<\Delta<1} \sup _{t_{0} \leq t \leq T} \mathbb{E}_{W}\left|\bar{x}_{\Delta}(t)\right|^{p} \leq C
$$

\section{Main results on classical SDEs}

In this section, the strong convergence of the truncated EM method is proved and the convergence rate is given. The main theorem of this paper is as follows.

Theorem 3.1. Let Assumptions 2.1, 2.2 and 2.5 hold. In addition, assume that (3) in Assumption 2.3 is true for any $p>2$. Then for any $\bar{q} \geq 2, \Delta \in(0,1]$ and any $\varepsilon \in(0,1 / 4)$,

$$
\sup _{t_{0} \leq t \leq T} \mathbb{E}_{W}\left|x(t)-x_{\Delta}(t)\right|^{\bar{q}} \leq C \Delta^{\min \left(\gamma, \alpha, \frac{1}{2}-\varepsilon\right) \bar{q}},
$$

and

$$
\sup _{t_{0} \leq t \leq T} \mathbb{E}_{W}\left|x(t)-\bar{x}_{\Delta}(t)\right|^{\bar{q}} \leq C \Delta^{\min \left(\gamma, \alpha, \frac{1}{2}-\varepsilon\right) \bar{q}}
$$


Remark 3.2. To obtain the results hold for any $\bar{q} \geq 2$ and arbitrarily $\varepsilon \in$ $(0,1 / 4)$, Assumption 2.3 is strengthened by requiring (3) to hold for any $p>2$ instead of some $p>2$ in Theorem 3.1. In this circumstance, the $L_{2}$ in (3) is no longer dependent on $p$.

To prove Theorem 3.1, we show Theorem 3.3 firstly, in which the format of the convergence rate is a bit complicated. The proof of Theorem 3.1 is postponed after the proof of the following theorem.

It should be noted that in Theorem 3.3 the Assumption 2.3 is not required to be strengthened compared with Theorem 3.1.

Theorem 3.3. Let Assumptions 2.1, 2.2, 2.3 and 2.5 hold with $p>(1+\beta) q$. Then, for any $\bar{q} \in[2, q)$ and $\Delta \in(0,1]$

$\mathbb{E}_{W}\left|x(t)-x_{\Delta}(t)\right|^{\bar{q}} \leq C\left(\left(f^{-1}(\kappa(\Delta))\right)^{[(1+\beta) \bar{q}-p]}+\Delta^{\bar{q} / 2}(\kappa(\Delta))^{\bar{q}}+\Delta^{\gamma \bar{q}}+\Delta^{\alpha \bar{q}}\right)$

and

$\mathbb{E}_{W}\left|x(t)-\bar{x}_{\Delta}(t)\right|^{\bar{q}} \leq C\left(\left(f^{-1}(\kappa(\Delta))\right)^{[(1+\beta) \bar{q}-p]}+\Delta^{\bar{q} / 2}(\kappa(\Delta))^{\bar{q}}+\Delta^{\gamma \bar{q}}+\Delta^{\alpha \bar{q}}\right)$

Proof. Fix $\bar{q}=[2, q)$ and $\Delta \in(0,1]$ arbitrarily. Let $e_{\Delta}(t)=x(t)-x_{\Delta}(t)$ for $t \in\left[t_{0}, T\right]$. By the Itô formula, we have for any $t_{0} \leq t \leq T$,

$$
\begin{aligned}
\mathbb{E}_{W}\left|e_{\Delta}(t)\right|^{\bar{q}} \leq & \mathbb{E}_{W} \int_{t_{0}}^{t} \bar{q}\left|e_{\Delta}(s)\right|^{\bar{q}-2}\left(e_{\Delta}^{T}(s)\left[\mu(s, x(s))-\mu_{\Delta}\left(\tau(s), \bar{x}_{\Delta}(s)\right)\right]\right. \\
& \left.+\frac{\bar{q}-1}{2} \sum_{r=1}^{m}\left|\sigma^{r}(s, x(s))-\sigma_{\Delta}^{r}\left(\tau(s), \bar{x}_{\Delta}(s)\right)\right|^{2}\right) d s .
\end{aligned}
$$

By the Young inequality $2 a b \leq \varepsilon a^{2}+b^{2} / \varepsilon$ for any $a, b \geq 0$ and $\varepsilon$ arbitrary, 
choosing $\varepsilon=(q-\bar{q}) /(\bar{q}-1)$ leads to

$$
\begin{aligned}
& \frac{\bar{q}-1}{2} \sum_{r=1}^{m}\left|\sigma^{r}(s, x(s))-\sigma_{\Delta}^{r}\left(\tau(s), \bar{x}_{\Delta}(s)\right)\right|^{2} \\
\leq & \frac{\bar{q}-1}{2} \sum_{r=1}^{m}\left(\left(1+\frac{q-\bar{q}}{\bar{q}-1}\right)\left|\sigma^{r}(s, x(s))-\sigma^{r}\left(s, x_{\Delta}(s)\right)\right|^{2}\right. \\
& \left.+\left(1+\frac{\bar{q}-1}{q-\bar{q}}\right)\left|\sigma^{r}\left(s, x_{\Delta}(s)\right)-\sigma_{\Delta}^{r}\left(\tau(s), \bar{x}_{\Delta}(s)\right)\right|^{2}\right) \\
= & \frac{q-1}{2} \sum_{r=1}^{m}\left|\sigma^{r}(s, x(s))-\sigma^{r}\left(s, x_{\Delta}(s)\right)\right|^{2} \\
+ & \frac{(\bar{q}-1)(q-1)}{2(q-\bar{q})} \sum_{r=1}^{m}\left|\sigma^{r}\left(s, x_{\Delta}(s)\right)-\sigma_{\Delta}^{r}\left(\tau(s), \bar{x}_{\Delta}(s)\right)\right|^{2} .
\end{aligned}
$$

$140 \quad$ We can get from (7) that

$$
\begin{aligned}
& \mathbb{E}_{W}\left|e_{\Delta}(t)\right|^{\bar{q}} \\
\leq & \mathbb{E}_{W} \int_{t_{0}}^{t} \bar{q}\left|e_{\Delta}(s)\right|^{\bar{q}-2}\left(e_{\Delta}^{T}(s)\left[\mu(s, x(s))-\mu\left(s, x_{\Delta}(s)\right)\right]\right. \\
& \left.+\frac{q-1}{2} \sum_{r=1}^{m}\left[\sigma^{r}(s, x(s))-\sigma^{r}\left(s, x_{\Delta}(s)\right)\right]^{2}\right) \mathrm{d} s \\
& +\mathbb{E}_{W} \int_{t_{0}}^{t} \bar{q}\left|e_{\Delta}(s)\right|^{\bar{q}-2} e_{\Delta}^{T}(t)\left[\mu\left(s, x_{\Delta}(s)\right)-\mu\left(\tau(s), x_{\Delta}(s)\right)\right] \mathrm{d} s \\
& +\mathbb{E}_{W} \int_{t_{0}}^{t} \bar{q}\left|e_{\Delta}(s)\right|^{\bar{q}-2} e_{\Delta}^{T}(t)\left[\mu\left(\tau(s), x_{\Delta}(s)\right)-\mu_{\Delta}\left(\tau(s), \bar{x}_{\Delta}(s)\right)\right] \mathrm{d} s \\
& +\mathbb{E}_{W} \int_{t_{0}}^{t} \bar{q}\left|e_{\Delta}(s)\right|^{\bar{q}-2} \frac{(\bar{q}-1)(q-1)}{(q-\bar{q})} \sum_{r=1}^{m}\left|\sigma^{r}\left(s, x_{\Delta}(s)\right)-\sigma^{r}\left(\tau(s), x_{\Delta}(s)\right)\right|^{2} \mathrm{~d} s \\
& +\mathbb{E}_{W} \int_{t_{0}}^{t} \bar{q}\left|e_{\Delta}(s)\right|^{\bar{q}-2} \frac{(\bar{q}-1)(q-1)}{(q-\bar{q})} \sum_{r=1}^{m}\left|\sigma^{r}\left(\tau(s), x_{\Delta}(s)\right)-\sigma_{\Delta}^{r}\left(\tau(s), \bar{x}_{\Delta}(s)\right)\right|^{2} \mathrm{~d} s .
\end{aligned}
$$

This implies

$$
\mathbb{E}_{W}\left|e_{\Delta}(t)\right|^{\bar{q}} \leq I_{1}+I_{2}+I_{3},
$$

141 where

$$
\begin{aligned}
I_{1}= & \mathbb{E}_{W} \int_{t_{0}}^{t} \bar{q}\left|e_{\Delta}(s)\right|^{\bar{q}-2}\left(e_{\Delta}^{T}(s)\left[\mu(s, x(s))-\mu\left(s, x_{\Delta}(s)\right)\right]\right. \\
& \left.+\frac{q-1}{2} \sum_{r=1}^{m}\left[\sigma^{r}(s, x(s))-\sigma^{r}\left(s, x_{\Delta}(s)\right)\right]^{2}\right) \mathrm{d} s
\end{aligned}
$$




$$
\begin{aligned}
I_{2}= & \mathbb{E}_{W} \int_{t_{0}}^{t} \bar{q}\left|e_{\Delta}(s)\right|^{\bar{q}-2}\left(e_{\Delta}^{T}(s)\left[\mu\left(s, x_{\Delta}(s)\right)-\mu\left(\tau(s), x_{\Delta}(s)\right)\right]\right. \\
& \left.+\frac{(\bar{q}-1)(q-1)}{(q-\bar{q})} \sum_{r=1}^{m}\left|\sigma^{r}\left(s, x_{\Delta}(s)\right)-\sigma^{r}\left(\tau(s), x_{\Delta}(s)\right)\right|^{2}\right) \mathrm{d} s,
\end{aligned}
$$

142 and

$$
\begin{aligned}
I_{3}= & \mathbb{E}_{W} \int_{t_{0}}^{t} \bar{q}\left|e_{\Delta}(s)\right|^{\bar{q}-2}\left(e_{\Delta}^{T}(s)\left[\mu\left(\tau(s), x_{\Delta}(s)\right)-\mu_{\Delta}\left(\tau(s), \bar{x}_{\Delta}(s)\right)\right]\right. \\
& \left.+\frac{(\bar{q}-1)(q-1)}{(q-\bar{q})} \sum_{r=1}^{m}\left|\sigma^{r}\left(\tau(s), x_{\Delta}(s)\right)-\sigma_{\Delta}^{r}\left(\tau(s), \bar{x}_{\Delta}(s)\right)\right|^{2}\right) \mathrm{d} s .
\end{aligned}
$$

By Assumption 2.2, we have

$$
I_{1} \leq C_{1} \mathbb{E}_{W} \int_{t_{0}}^{t}\left|e_{\Delta}(s)\right|^{\bar{q}} \mathrm{~d} s
$$

where $C_{1}=L_{1} \bar{q}$. Using the Young inequality and Assumption 2.5, we can derive

$$
\begin{aligned}
I_{2} \leq & \mathbb{E}_{W} \int_{t_{0}}^{t} \bar{q}\left|e_{\Delta}(s)\right|^{\bar{q}-2}\left(\frac{1}{2}\left|e_{\Delta}(s)\right|^{2}+\frac{1}{2}\left|\mu\left(s, x_{\Delta}(s)\right)-\mu\left(\tau(s), x_{\Delta}(s)\right)\right|^{2}\right. \\
& \left.+\frac{(\bar{q}-1)(q-1)}{(q-\bar{q})} \sum_{r=1}^{m}\left|\sigma^{r}\left(s, x_{\Delta}(s)\right)-\sigma^{r}\left(\tau(s), x_{\Delta}(s)\right)\right|^{2}\right) \mathrm{d} s \\
\leq & C_{2}\left(\mathbb{E}_{W} \int_{t_{0}}^{t}\left|e_{\Delta}(s)\right|^{\bar{q}} d s+\mathbb{E}_{W} \int_{t_{0}}^{t}\left|\mu\left(s, x_{\Delta}(s)\right)-\mu\left(\tau(s), x_{\Delta}(s)\right)\right|^{\bar{q}} \mathrm{~d} s\right. \\
& \left.+\frac{2(\bar{q}-1)(q-1)}{(q-\bar{q})} \sum_{r=1}^{m} \mathbb{E}_{W} \int_{t_{0}}^{t}\left|\sigma^{r}\left(s, x_{\Delta}(s)\right)-\sigma^{r}\left(\tau(s), x_{\Delta}(s)\right)\right|^{\bar{q}} \mathrm{~d} s\right) \\
\leq & C_{2}\left(\mathbb{E}_{W} \int_{t_{0}}^{t}\left|e_{\Delta}(s)\right|^{\bar{q}} d s+\mathbb{E}_{W} \int_{t_{0}}^{t} K_{1}^{\bar{q}}\left(1+\left|x_{\Delta}(s)\right|^{(\beta+1) \bar{q}}\right) \Delta^{\gamma \bar{q}} \mathrm{~d} s\right. \\
& \left.+\mathbb{E}_{W} \int_{t_{0}}^{t} K_{2}^{\bar{q}}\left(1+\left|x_{\Delta}(s)\right|^{(\beta+1) \bar{q}}\right) \Delta^{\alpha \bar{q}} \mathrm{~d} s\right) .
\end{aligned}
$$

Then by Lemma 2.8, we obtain

$$
I_{2} \leq C_{2}\left(\mathbb{E}_{W} \int_{t_{0}}^{t}\left|e_{\Delta}(s)\right|^{\bar{q}} \mathrm{~d} s+\Delta^{\gamma \bar{q}}+\Delta^{\alpha \bar{q}}\right) .
$$


144

Rearranging $I_{3}$ gives

$$
\begin{aligned}
I_{3} \leq & \mathbb{E}_{W} \int_{t_{0}}^{t} \bar{q}\left|e_{\Delta}(s)\right|^{\bar{q}-2}\left(e_{\Delta}^{T}(t)\left[\mu\left(\tau(s), x_{\Delta}(s)\right)-\mu\left(\tau(s), \bar{x}_{\Delta}(s)\right)\right]\right. \\
& \left.+\frac{2(\bar{q}-1)(q-1)}{(q-\bar{q})} \sum_{r=1}^{m}\left|\sigma^{r}\left(\tau(s), x_{\Delta}(s)\right)-\sigma^{r}\left(\tau(s), \bar{x}_{\Delta}(s)\right)\right|^{2}\right) \mathrm{d} s \\
& +\mathbb{E}_{W} \int_{t_{0}}^{t} \bar{q}\left|e_{\Delta}(s)\right|^{\bar{q}-2}\left(e_{\Delta}^{T}(t)\left[\mu\left(\tau(s), \bar{x}_{\Delta}(s)\right)-\mu_{\Delta}\left(\tau(s), \bar{x}_{\Delta}(s)\right)\right]\right. \\
& \left.+\frac{2(\bar{q}-1)(q-1)}{(q-\bar{q})} \sum_{r=1}^{m}\left|\sigma^{r}\left(\tau(s), \bar{x}_{\Delta}(s)\right)-\sigma_{\Delta}^{r}\left(\tau(s), \bar{x}_{\Delta}(s)\right)\right|^{2}\right) \mathrm{d} s \\
:= & I_{31}+I_{32} .
\end{aligned}
$$

$$
\begin{aligned}
I_{32} \leq & C_{32}\left(\mathbb{E}_{W} \int_{t_{0}}^{t}\left|e_{\Delta}(s)\right|^{\bar{q}} \mathrm{~d} s+\mathbb{E}_{W} \int_{t_{0}}^{t}\left|\mu\left(\tau(s), \bar{x}_{\Delta}(s)\right)-\mu_{\Delta}\left(\tau(s), \bar{x}_{\Delta}(s)\right)\right|^{\bar{q}}\right. \\
& \left.+\sum_{r=1}^{m}\left|\sigma^{r}\left(\tau(s), \bar{x}_{\Delta}(s)\right)-\sigma_{\Delta}^{r}\left(\tau(s), \bar{x}_{\Delta}(s)\right)\right|^{\bar{q}} \mathrm{~d} s\right) .
\end{aligned}
$$

Then, by the Hölder inequality, Lemma 2.7 and Lemma 2.8, we arrive at

$$
\begin{aligned}
I_{31} & \leq C_{31}\left(\mathbb{E}_{W} \int_{t_{0}}^{t}\left|e_{\Delta}(s)\right|^{\bar{q}} \mathrm{~d} s+\int_{t_{0}}^{t}\left(\mathbb{E}_{W}\left|x_{\Delta}(s)-\bar{x}_{\Delta}(s)\right|^{p}\right)^{\frac{\bar{q}}{p}} \mathrm{~d} s\right) \\
& \leq C_{31}\left(\mathbb{E}_{W} \int_{t_{0}}^{t}\left|e_{\Delta}(s)\right|^{\bar{q}} \mathrm{~d} s+\Delta^{\frac{\bar{q}}{2}}(\kappa(\Delta))^{\bar{q}} \mathrm{~d} s\right) .
\end{aligned}
$$




$$
\begin{aligned}
& I_{32} \leq C_{32}\left(\mathbb{E}_{W} \int_{t_{0}}^{t}\left|e_{\Delta}(s)\right|^{\bar{q}} \mathrm{~d} s+\int_{t_{0}}^{t}\left[\mathbb{E}_{W}\left(1+\left|\bar{x}_{\Delta}(s)\right|^{p}+\left|\pi_{\Delta}\left(\bar{x}_{\Delta}(s)\right)\right|^{p}\right)\right]^{\frac{\beta \bar{q}}{p}}\right. \\
& \left.\times\left(\mathbb{E}_{W} \mid \bar{x}_{\Delta}(s)-\pi_{\Delta}\left(\bar{x}_{\Delta}(s)\right)^{\frac{p \bar{q}}{p-\beta \bar{q}}}\right)^{\frac{p-\beta \bar{q}}{p}} \mathrm{~d} s\right) \\
& \leq C_{32}\left(\mathbb{E}_{W} \int_{t_{0}}^{t}\left|e_{\Delta}(s)\right|^{\bar{q}} \mathrm{~d} s+\int_{t_{0}}^{t}\left(\mathbb{E}_{W}\left[I_{\left\{\left|\bar{x}_{\Delta}(s)\right|>f^{-1}(\kappa(\Delta))\right\}}\left|x_{\Delta}(s)\right|^{\frac{p \bar{p}}{p-\beta \bar{q}}}\right]\right)^{\frac{p-\beta \bar{q}}{p}} \mathrm{~d} s\right) \\
& \leq C_{32}\left(\mathbb{E}_{W} \int_{t_{0}}^{t}\left|e_{\Delta}(s)\right|^{\bar{q}} \mathrm{~d} s\right. \\
& \left.\left.+\int_{t_{0}}^{t}\left(\left.\left[\mathbb{P}\left\{\left|\bar{x}_{\Delta}(s)\right|>f^{-1}(\kappa(\Delta))\right\}\right]^{\frac{p-\beta \bar{q}-\bar{q}}{p-\beta \bar{q}}}\left[\mathbb{E}_{W} \mid \bar{x}_{\Delta}(s)\right)\right|^{p}\right]^{\frac{\bar{q}}{p-\beta \bar{q}}}\right)^{\frac{p-\beta \bar{q}}{p}} \mathrm{~d} s\right) \\
& \leq C_{32}\left(\mathbb{E}_{W} \int_{t_{0}}^{t}\left|e_{\Delta}(s)\right|^{\bar{q}} \mathrm{~d} s+\int_{t_{0}}^{T}\left(\frac{\mathbb{E}_{W}\left|\bar{x}_{\Delta}(s)\right|^{p}}{\left(f^{-1}(\kappa(\Delta))\right)^{p}}\right)^{\frac{p-\beta \bar{q}-\bar{q}}{p}} \mathrm{~d} s\right) \\
& \leq C_{32}\left(\mathbb{E}_{W} \int_{t_{0}}^{t}\left|e_{\Delta}(s)\right|^{\bar{q}} \mathrm{~d} s+\left(f^{-1}(\kappa(\Delta))\right)^{(\beta+1) \bar{q}-p}\right) \text {. }
\end{aligned}
$$

Substituting (11) and (12) into (10), we arrive at

$$
I_{3} \leq C_{3}\left(\mathbb{E}_{W} \int_{t_{0}}^{t}\left|e_{\Delta}(s)\right|^{\bar{q}} \mathrm{~d} s+\Delta^{\frac{\bar{q}}{2}}(\kappa(\Delta))^{\bar{q}}+\left(f^{-1}(\kappa(\Delta))\right)^{(\beta+1) \bar{q}-p}\right) .
$$

Then (8), (9) and (13) together imply that

$$
\mathbb{E}_{W}\left|e_{\Delta}(t)\right|^{\bar{q}} \leq C\left(\mathbb{E}_{W} \int_{t_{0}}^{t}\left|e_{\Delta}(s)\right|^{\bar{q}} \mathrm{~d} s+\left(f^{-1}(\kappa(\Delta))\right)^{(\beta+1) \bar{q}-p}+\Delta^{\frac{\bar{q}}{2}}(\kappa(\Delta))^{\bar{q}}+\Delta^{\gamma \bar{q}}+\Delta^{\alpha \bar{q}}\right) .
$$

An application of the Gronwall inequality yields that

$$
\mathbb{E}_{W}\left|e_{\Delta}(t)\right|^{\bar{q}} \leq C\left(\left(f^{-1}(\kappa(\Delta))\right)^{(\beta+1) \bar{q}-p}+\Delta^{\frac{\bar{q}}{2}}(\kappa(\Delta))^{\bar{q}}+\Delta^{\gamma \bar{q}}+\Delta^{\alpha \bar{q}}\right),
$$


Proof of Theorem 3.1

Recalling (2), we then define

$$
f(u)=K u^{\beta+2}, u \geq 1,
$$

which implies that

$$
f^{-1}(u)=\left(\frac{u}{K}\right)^{\frac{1}{\beta+2}}
$$

Let

$$
\kappa(\Delta)=\Delta^{-\varepsilon} \text { for some } \varepsilon \in\left(0, \frac{1}{4}\right) \text { and } \hat{h} \geq 1 .
$$

Following Theorem 3.3, we obtain

$$
\mathbb{E}_{W}\left|x(t)-x_{\Delta}(t)\right|^{\bar{q}} \leq C\left(\Delta^{\frac{\varepsilon(p-\beta \bar{q}-\bar{q})}{\beta+2}}+\Delta^{\frac{\bar{q}(1-2 \varepsilon)}{2}}+\Delta^{\gamma \bar{q}}+\Delta^{\alpha \bar{q}}\right),
$$

and

$$
\mathbb{E}_{W}\left|x(t)-\bar{x}_{\Delta}(t)\right|^{\bar{q}} \leq C\left(\Delta^{\frac{\varepsilon(p-\beta \bar{q}-\bar{q})}{\beta+2}}+\Delta^{\frac{\bar{q}(1-2 \varepsilon)}{2}}+\Delta^{\gamma \bar{q}}+\Delta^{\alpha \bar{q}}\right) .
$$

Choosing $p$ sufficiently large for

$$
\frac{\varepsilon(p-\beta \bar{q}-\bar{q})}{\beta+2}>\min \left(\gamma, \alpha, \frac{1}{2}-\varepsilon\right) \bar{q},
$$

we can draw the assertions from (14) and (15) immediately.

\section{Main results on time-changed SDEs}

This section is divided into two parts. In Section 4.1, mathematical preliminaries about time-changed SDEs are presented together with some useful lemmas. The result on the strong convergence of the truncated EM method is presented in Section 4.2 . 
Let $D(t)$ be a right continuous with the existence of the left limit increasing Lévy process defined on a complete probability space $\left(\Omega_{D}, \mathcal{F}^{D}, \mathbb{P}_{D}\right)$ with a filtration $\left\{\mathcal{F}_{t}^{D}\right\}_{t \geq 0}$ satisfying the usual conditions. Let $\mathbb{E}_{D}$ denote the expectation under the probability measure $\mathbb{P}_{D} . D(t)$ is called subordinator starting from 0 if the Laplace transform is given by

$$
\mathbb{E}_{D} e^{-\lambda D(t)}=e^{-t \phi(\lambda)},
$$

where the Laplace exponent is

$$
\phi(\lambda)=\int_{0}^{\infty}\left(1-e^{-\lambda x}\right) \nu(d x),
$$

with $\int_{0}^{\infty}(x \wedge 1) \nu(d x)<\infty$. We focus on the case when the Lévy measure $\nu$ is infinity, i.e. $\nu(0, \infty)=\infty$., which implies that $D(t)$ has strictly increasing paths with infinitely many jumps and excludes the compound Poisson subordinator.

Let $E(t)$ be the inverse of $D(t)$, i.e.

$$
E(t):=\inf \{u>0 ; D(u)>t\}, t \geq 0 .
$$

We call $E(t)$ an inverse subordinator.

Assume that $W(t)$ and $D(t)$ are independent. Define the product probability space by

$$
(\Omega, \mathcal{F}, \mathbb{P}):=\left(\Omega_{W} \times \Omega_{D}, \mathcal{F}^{W} \otimes \mathcal{F}^{D}, \mathbb{P}_{W} \otimes \mathbb{P}_{D}\right) .
$$

Let $\mathbb{E}$ denote the expectation under the probability measure $\mathbb{P}$. It is clear that $\mathbb{E}(\cdot)=\mathbb{E}_{D} \mathbb{E}_{W}(\cdot)=\mathbb{E}_{W} \mathbb{E}_{D}(\cdot)$.

In this section, we consider the following time-changed SDE

$$
d y(t)=\mu(E(t), y(t)) d E(t)+\sigma(E(t), y(t)) d W(E(t)), t \in[0, T],
$$

with the initial value $y(0)=y_{0}$. Here, for the simplicity of the notation, we only consider the scale Wiener process $W$ (i.e. $m=1$ in Sections 2 and 3).

According to the duality principle, the time-changed SDE (16) and the classical SDE of Itô type

$$
d x(t)=\mu(t, x(t)) d t+\sigma(t, x(t)) d W(t)
$$


have a deep connection. The next theorem states such a relation more precisely, which is borrowed from Theorem 4.2 in [18].

Theorem 4.1. Suppose Assumptions 2.1, 2.2, 2.3 and 2.5 hold. If $x(t)$ is the unique solution to the SDE (17), then the time-changed process $x(E(t))$, which is an $\mathcal{F}_{E(t)}^{W}$-semimartingale, is the unique solution to the time-changed SDE (16). On the other hand, if $y(t)$ is the unique solution to the time-changed $S D E$ (16), then the process $y(D(t))$, which is an $\mathcal{F}_{t}^{W}$-semimartingale, is the unique solution to the SDE (17).

The plan to numerically approximate the time-changed SDE (16) in this section is as follows. Firstly, we discretize the inverse subordinator $E(t)$ to get $E_{\Delta}(t)$. Then the combination, $x_{\Delta}\left(E_{\Delta}(t)\right)$, of the truncated EM solution to the $\operatorname{SDE}(17), x_{\Delta}(t)$, and the discretized inverse subordinator, $E_{\Delta}(t)$, is used to approximate the solution to the time-changed SDE (16).

To approximate the $E(t)$ in a given time interval $[0, T]$, we follow the idea in [6]. Firstly, we simulate the path of $D(t)$ by $D_{\Delta}\left(t_{i}\right)=D_{\Delta}\left(t_{i-1}\right)+\xi_{i}$ with $D\left(t_{0}\right)=0$ and $t_{i}=t_{0}+i \Delta$, where $\xi_{i}$ is independently identically sequence with $\xi_{i}=D\left(t_{1}\right)-D\left(t_{0}\right)$ in distribution. The process is stopped when

$$
T \in\left[D_{\Delta}\left(t_{n}\right), D_{\Delta}\left(t_{n+1}\right)\right)
$$

for some $n$. Then the approximate $E_{\Delta}(t)$ to $E(t)$ is generated by

$$
E_{\Delta}(t)=\left(\min \left\{n ; D_{\Delta}\left(t_{n}\right)>t\right\}-1\right) \Delta
$$

for $t \in[0, T]$. It is easy to see

$$
E_{\Delta}(t)=i \Delta, \quad \text { when } t \in\left[D_{\Delta}\left(t_{i}\right), D_{\Delta}\left(t_{i+1}\right)\right)
$$

It is not hard to observe that $E_{\Delta}(t)$ leads to the uniform partition in the timechanged case as well. The next lemma provides the approximation error of $E_{\Delta}(t)$ to $E(t)$, whose proof can be found in Theorem 2 in [21].

Lemma 4.2. Let $E(t)$ be the inverse of a subordinator $D(t)$ with infinite Lévy measure. Then for any $t \in[0, T]$

$$
E(t)-\Delta \leq E_{\Delta}(t) \leq E(t) \quad \text { a.s. }
$$


The following lemma states that any inverse subordinator $E(t)$ with infinite Lévy measure is known to have the exponential moment, see for example Lemma 2.7 in [4].

Lemma 4.3. Let $E(t)$ be the inverse of a subordinator $D(t)$ with Laplace exponent $\phi$ and infinite Lévy measure, then for any $C \in \mathbb{R}$ and $t \geq 0$,

$$
\mathbb{E}_{D}\left(e^{C E(t)}\right)<\infty .
$$

We also need the continuity of the solution to (17) presented in the next lemma. The proof is not hard to obtain by using the standard approach (see for example Theorem 4.3 in Page 61 of [23]).

Lemma 4.4. Suppose that Assumptions 2.1 and 2.3 hold. Then for any $q<$ $p /(\beta+1)$ and $|t-s|<1$, the solution to (17) satisfies

$$
\mathbb{E}_{W}|x(t)-x(s)|^{q} \leq C_{4}|t-s|^{q / 2} e^{C_{4} t},
$$

where $C_{4}$ is a constant independent of $t$ and $s$.

\subsection{Strong convergence of the truncated EM method for time-changed SDEs}

Before the main result is presented, we make some remarks on the constant, $C$, in Theorem 3.1. Since the main purpose of Theorem 3.1 is to show the convergence rate, we do not give the explicit form of the constant $C$. But it is not hard by going through the proof to see that $C(t):=C$ contains the time variable $t$ only in the form of $\exp ($ some constant $\times t)$. This means that if we replace $t$ by $E(t)$, we have $\mathbb{E}_{D}(C(E(t)))<\infty$ by Lemma 4.3 .

Theorem 4.5. Let Assumptions 2.1, 2.2 and 2.5 hold. In addition, assume that (3) in Assumption 2.3 is true for any $p>2$. Then the combination of the truncated EM solution and the discretized inverse subordinator, i.e. $x_{\Delta}\left(E_{\Delta}(t)\right)$, converges strongly to the solution of (16)

$$
\mathbb{E}\left|y(t)-x_{\Delta}\left(E_{\Delta}(t)\right)\right|^{\bar{q}} \leq C_{t c} \Delta^{\min \left(\gamma, \alpha, \frac{1}{2}-\varepsilon\right) \bar{q}},
$$

for any $\bar{q} \geq 2, \Delta \in(0,1], \varepsilon \in(0,1 / 4)$ and $t \in[0, T]$, where $C_{t c}$ is constant independent from $\Delta$. 
Proof. By Theorem 4.1 and the elementary inequality, we have

$$
\begin{aligned}
& \mathbb{E}\left|y(t)-x_{\Delta}\left(E_{\Delta}(t)\right)\right|^{\bar{q}} \\
= & \mathbb{E}\left|x(E(t))-x_{\Delta}\left(E_{\Delta}(t)\right)\right|^{\bar{q}} \\
\leq & 2^{\bar{q}-1}\left(\mathbb{E}\left|x(E(t))-x\left(E_{\Delta}(t)\right)\right|^{\bar{q}}+\mathbb{E} \mid x\left(E_{\Delta}(t)-\left.x_{\Delta}\left(E_{\Delta}(t)\right)\right|^{\bar{q}}\right) .\right.
\end{aligned}
$$

By Lemmas 4.2, 4.3 and 4.4, we can see

$$
\mathbb{E}\left|x(E(t))-x\left(E_{\Delta}(t)\right)\right|^{\bar{q}} \leq C_{4} \Delta^{\bar{q} / 2} \mathbb{E}_{D}\left(e^{C_{4} E(t)}\right) \leq C_{5} \Delta^{\bar{q} / 2},
$$

where $C_{5}$ is a constant independent from $\Delta$. By Lemma 4.2 and Theorem 3.1, we obtain

$$
\mathbb{E} \mid x\left(E_{\Delta}(t)-\left.x_{\Delta}\left(E_{\Delta}(t)\right)\right|^{\bar{q}} \leq \mathbb{E}_{D}(C) \Delta^{\min \left(\gamma, \alpha, \frac{1}{2}-\varepsilon\right) \bar{q}} \leq C_{6} \Delta^{\min \left(\gamma, \alpha, \frac{1}{2}-\varepsilon\right) \bar{q}}\right.
$$

where $C_{6}$ is a constant independent from $\Delta$. Combining (19) and (20), we have the required assertion.

\section{Numerical Simulations}

This section is divided into two parts. The numerical simulations on SDEs are presented in Section 5.1 and time-changed SDEs are displayed in Section 5.2 .

\subsection{Simulations for SDEs}

Two examples with the different theoretical convergence rates are presented in this part. Computer simulations are conducted to verify the theoretical results.

Example 5.1. Consider a scalar stochastic differential equation

$$
\left\{\begin{array}{l}
\mathrm{d} x(t)=\left([t(1-t)]^{\frac{1}{4}} x^{2}(t)-2 x^{5}(t)\right) \mathrm{d} t+\left([t(1-t)]^{\frac{1}{4}} x^{2}(t)\right) \mathrm{d} W(t), \\
x\left(t_{0}\right)=2
\end{array}\right.
$$


For any $q>2$, we can see

$$
\begin{aligned}
& (x-y)^{T}(\mu(t, x)-\mu(t, y))+\frac{q-1}{2}\left|\sigma^{r}(t, x)-\sigma^{r}(t, y)\right|^{2} \\
\leq & (x-y)^{2}\left([t(1-t)]^{\frac{1}{4}}(x+y)-2\left(x^{4}+x^{3} y+x^{2} y^{2}+x y^{3}+y^{4}\right)\right. \\
& \left.+\frac{q-1}{2}[t(1-t)]^{\frac{1}{2}}(x+y)^{2}\right) .
\end{aligned}
$$

But

$$
-2\left(x^{3} y+x y^{3}\right)=-2 x y\left(x^{2}+y^{2}\right) \leq\left(x^{2}+y^{2}\right)^{2}=x^{4}+y^{4}+2 x^{2} y^{2} .
$$

and

$$
\mathbb{E}_{W}\left|x(t)-\bar{x}_{\Delta}(t)\right|^{\bar{q}} \leq C\left(\left(f^{-1}(\kappa(\Delta))\right)^{(5 \bar{q}-p)}+\Delta^{\bar{q} / 2}(\kappa(\Delta))^{\bar{q}}+\Delta^{\bar{q} / 4}\right) .
$$


Due to that

$$
\sup _{t_{0} \leq t \leq T} \sup _{|x| \leq u}(|\mu(t, x) \vee| \sigma(t, x) \mid) \leq 3 u^{5}, \quad \forall u \geq 1,
$$

we choose $f(u)=3 u^{5}$ and $\kappa(\Delta)=\Delta^{-\varepsilon}$, for any $\varepsilon \in(0,1 / 4)$. As a result, $f^{-1}(u)=(u / 3)^{1 / 5}$ and $f^{-1}(\kappa(\Delta))=\left(\Delta^{-\varepsilon} / 3\right)^{1 / 5}$. Choosing $p$ sufficiently large, we can get from Theorem 3.1 that

$$
\sup _{0 \leq t \leq 1} \mathbb{E}_{W}\left|x(t)-x_{\Delta}(t)\right|^{\bar{q}} \leq C \Delta^{\bar{q} / 4}
$$

and

$$
\sup _{0 \leq t \leq 1} \mathbb{E}_{W}\left|x(t)-\bar{x}_{\Delta}(t)\right|^{\bar{q}} \leq C \Delta^{\bar{q} / 4},
$$

which imply that the convergence rate of truncated EM method for the SDE (21) is $1 / 4$.

Let us compute the approximation of the mean square error. We run $M=$ 1000 independent trajectories for every different step sizes, $10^{-1}, 10^{-2}, 10^{-3}$, $10^{-8}$. Because it is hard to find the true solution for the SDE, the numerical solution with the step size $10^{-8}$ is regarded as the exact solution.

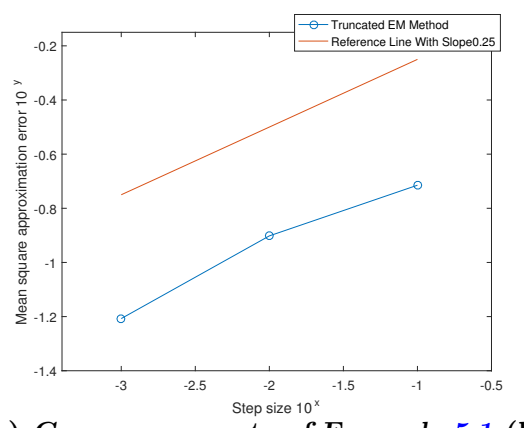

(a) Convergence rate of Example 5.1 (b)

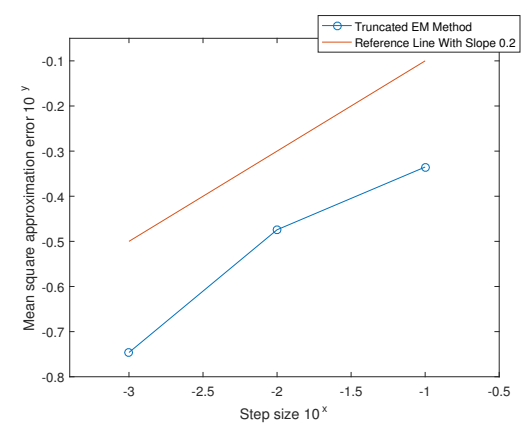

b) Convergence rate of Example 5.2

Figure 1: The $L^{1}$ errors between the exact solution and the numerical solutions for step sizes $\Delta=10^{-1}, 10^{-2}, 10^{-3}$.

By the linear regression, also shown in the Figure 1(a), the slope of the errors against the step sizes is approximately 0.24629 , which is quite close to the theoretical result. 
Example 5.2. Consider the scalar stochastic differential equation

$$
\left\{\begin{array}{l}
\mathrm{d} x(t)=\left([(t-1)(2-t)]^{\frac{1}{5}} x^{2}(t)-2 x^{5}(t)\right) \mathrm{d} t+\left([(t-1)(2-t)]^{\frac{2}{5}} x^{2}(t)\right) \mathrm{d} W(t), \\
x\left(t_{0}\right)=2
\end{array}\right.
$$

where $t_{0}=1$ and $T=2$. In the similar way as Example 5.1, we can verify that Assumptions 2.2 and 2.3 hold.

Moreover, the mean value theorem is used to verify that Assumptions 2.1 and 2.5 are satisfied with $\alpha=2 / 5, \gamma=1 / 5$ and $\beta=4$.

We can get from Theorem 3.1 that

$$
\sup _{1 \leq t \leq 2} \mathbb{E}_{W}\left|x(t)-x_{\Delta}(t)\right|^{\bar{q}} \leq C \Delta^{\bar{q} / 5},
$$

and

$$
\sup _{1 \leq t \leq 2} \mathbb{E}_{W}\left|x(t)-\bar{x}_{\Delta}(t)\right|^{\bar{q}} \leq C \Delta^{\bar{q} / 5},
$$

which implies that the convergence rate of truncated EM method for the SDE (22) is $1 / 5$. Simulation is conducted using the same strategy as that in Example 5.1. Using the linear regression, also seen in the figure 1(b), the slope of the errors against the step sizes is approximately 0.20550 , which coincides with the theoretical result.

\subsection{Simulations for time-changed SDEs}

Example 5.3. A two-dimensional time-changed SDE

$$
\left\{\begin{array}{l}
\mathrm{d} y_{1}(t)=-2 y_{1}^{4}(t) \mathrm{d} E(t)+y_{2}^{2}(t) \mathrm{d} W(E(t)) \\
\mathrm{d} y_{2}(t)=-2 y_{2}^{4}(t) \mathrm{d} E(t)+y_{1}^{2}(t) \mathrm{d} W(E(t))
\end{array}\right.
$$

is considered with the initial data $y_{1}(0)=1$ and $y_{2}(0)=2$.

For a given step size $h$, one path of the numerical solution to (23) is simulated in the following way. 
Step 1. The truncated EM method with the step size $\Delta$ is used to simulate the numerical solution, $x_{\Delta}\left(t_{k}\right)$, for $k=1,2,3, \ldots$, to the duel SDE

$$
\left\{\begin{array}{l}
\mathrm{d} x_{1}(t)=-2 x_{1}^{4}(t) \mathrm{d} t+x_{2}^{2}(t) \mathrm{d} W(t), \\
\mathrm{d} x_{2}(t)=-2 x_{2}^{4}(t) \mathrm{d} t+x_{1}^{2}(t) \mathrm{d} W(t) .
\end{array}\right.
$$

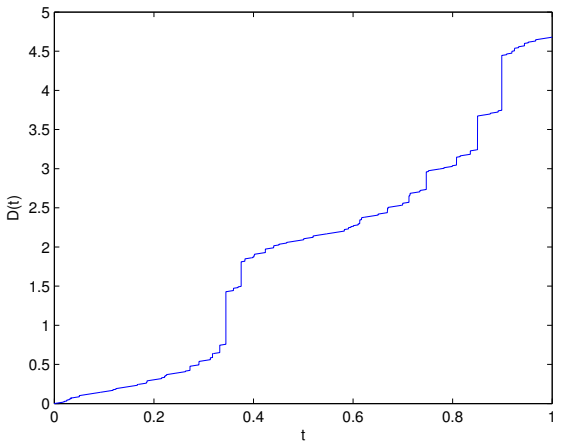

(a) One path of $D(t)$

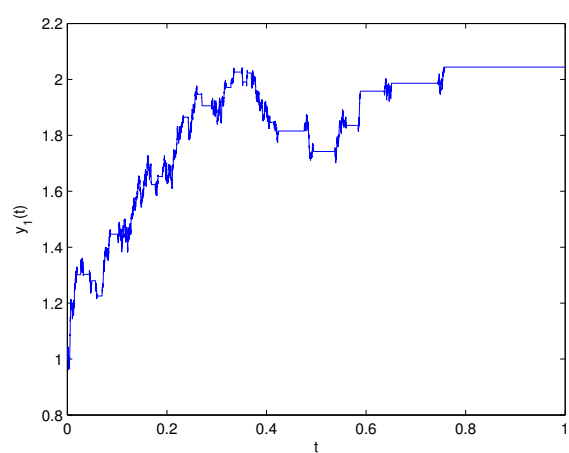

(c) One path of $y_{1}(t)$

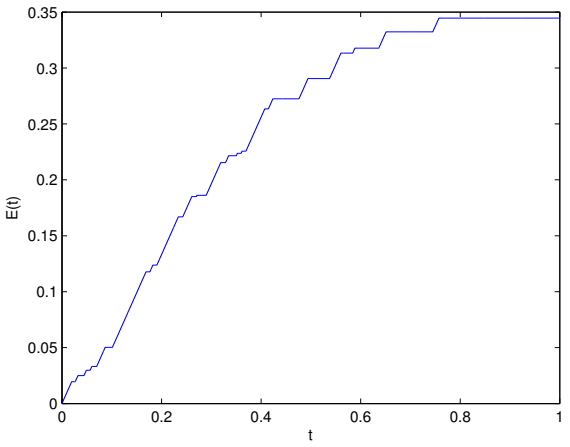

(b) One path of $E(t)$

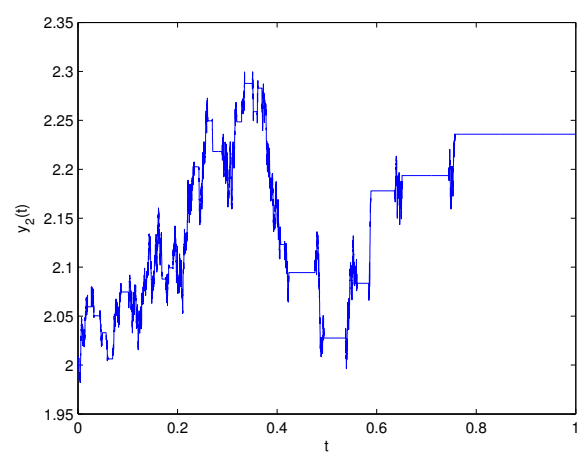

(d) One path of $y_{2}(t)$

Figure 2: Numerical simulations of $D(t), E(t), y_{1}(t)$ and $y_{2}(t)$ 


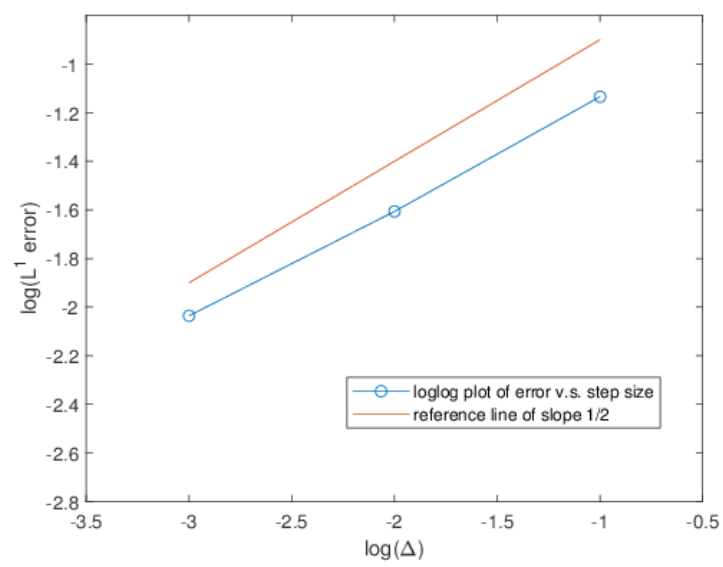

Figure 3: Blue line: Loglog plot of the strong $L^{1}$ error against the step size. Red Line: The reference line with the slope of $1 / 2$.

2(b) displays one path of $E(t)$. Paths of $y_{1}(t)$ and $y_{2}(t)$ are plotted in Figures $2(\mathrm{c})$ and $2(\mathrm{~d})$, respectively.

Now we demonstrate the strong convergence rate. Since the explicit solution ia hard to obtain, we treat the numerical solution with $\Delta=10^{-8}$ as the true solution. Two hundred samples are used to compute the strong convergence with the step sizes $10^{-1}, 10^{-2}$ and $10^{-3}$. We pick up $\epsilon=0.01$, by Theorem 4.5 a strong convergence rate that is closed to 0.5 is expected. The loglog plot of the $L^{1}$ errors against the step sizes is shown in Figure 3. By the linear regression, the slope is 0.4506 that is not far away from the theoretical result.

\section{Conclusion}

In this paper, we apply the truncated EM method for a class of non-autonomous classical SDEs with the Hölder continuity in the temporal variable and the super-linear growth in the state variable. The strong convergence with the rate is proved.

In addition, the results on the classical SDEs are used to prove that the truncated EM method can also work well for a class of highly non-linear time- 
changed SDEs. Such a result provides a trusted numerical method for a much larger class of time-changed SDEs than those in existing works.

\section{Acknowledgement}

Wei Liu is financially supported by the National Natural Science Foundation of China (11701378, 11871343, 11971316), "Chenguang Program" supported by both Shanghai Education Development Foundation and Shanghai Municipal Education Commission (16CG50), and Shanghai Gaofeng \& Gaoyuan Project for University Academic Program Development.

Xuerong Mao would like to thank the EPSRC (EP/K503174/1), the Royal Society (Wolfson Research Merit Award WM160014), the Royal Society and the Newton Fund (NA160317, Royal Society-Newton Advanced Fellowship) and the Ministry of Education (MOE) of China (MS2014DHDX020), for their financial support.

Yue Wu is financially supported by EPRSC EP/R041431/1.

\section{References}

\section{References}

[1] E. Allen, Modeling with Itô stochastic differential equations, volume 22 of Mathematical Modelling: Theory and Applications, Springer, Dordrecht, 2007.

[2] M. S. Alrawashdeh, J. F. Kelly, M. M. Meerschaert and H. Scheffler, Applications of inverse tempered stable subordinators, Comput. Math. Appl. 73 (6) (2017), 892-905.

[3] K. Dareiotis, C. Kumar and S. Sabanis, On tamed Euler approximations of SDEs driven by Lévy noise with applications to delay equations, SIAM J. Numer. Anal. 54 (3) (2016), 1840-1872. 
[4] C-S. Deng and W. Liu, Semi-implicit Euler-Maruyama method for a class of time-changed stochastic differential equations, Preprint, arXiv:1907.11408.

[5] C-S. Deng and R. L. Schilling, Harnack inequalities for SDEs driven by time-changed fractional Brownian motions, Electron. J. Probab. 22 (2017), Paper No. 71, 23 pp.

[6] J. Gajda and M. Magdziarz, Fractional Fokker-Planck equation with tempered $\alpha$-stable waiting times: Langevin picture and computer simulation, Phys. Rev. E 82 (1) (3) (2010), 011117, 6.

[7] Q. Guo, W. Liu, X. Mao and R. Yue, The partially truncated Euler-Maruyama method and its stability and boundedness, Appl. Numer. Math. 115 (2017), 235-251.

[8] Q. Guo, W. Liu, X. Mao and R. Yue, The truncated Milstein method for stochastic differential equations with commutative noise, J. Comput. Appl. Math. 338 (2018), 298-310.

[9] D.J. Higham, Stochastic ordinary differential equations in applied and computational mathematics, IMA J. Appl. Math. 76 (2011), 449-474. special issue: "2020 visions of applied mathematics".

[10] L. Hu, X. Li and X. Mao, Convergence rate and stability of the truncated Euler-Maruyama method for stochastic differential equations, J. Comput. Appl. Math. 337 (2018), 274-289.

[11] M. Hutzenthaler, A. Jentzen and P. E. Kloeden, Strong and weak divergence in finite time of Euler's method for stochastic differential equations with non-globally Lipschitz continuous coefficients, Proc. R. Soc. Lond. Ser. A Math. Phys. Eng. Sci. 467 (2011), 1563-1576.

[12] M. Hutzenthaler, A. Jentzen and P.E. Kloeden, Strong convergence of an explicit numerical method for SDEs with non-globally Lipschitz continuous coefficients, Ann. Appl. Probab. 22 (2) (2012), 1611-1641. 
[13] M. Hutzenthaler and A. Jentzen, Numerical approximations of stochastic differential equations with non-globally Lipschitz continuous coefficients, Mem. Amer. Math. Soc. 236 (2) (2015), 99 pages.

[14] S. Jin and K. Kobayashi, Strong approximation of stochastic differential equations driven by a time-changed brownian motion with time-spacedependent coefficients, J. Math. Anal. Appl. 476 (2) (2019), 619-636.

[15] E. Jum, Numerical Approximation of Stochastic Differential Equations Driven by Lévy Motion with Infinitely Many Jumps, PhD thesis, University of Tennessee - Knoxville, 2015.

[16] E. Jum and K. Kobayashi, A strong and weak approximation scheme for stochastic differential equations driven by a time-changed Brownian motion, Probab. Math. Statist. 36 (2) (2016), 201-220.

[17] P. E. Kloeden and E. Platen, Numerical solution of stochastic differential equations, volume 23 of Applications of Mathematics (New York), SpringerVerlag, Berlin, 1992.

[18] K. Kobayashi, Stochastic calculus for a time-changed semimartingale and the associated stochastic differential equations, J. Theoret. Probab. 24 (3) (2011), 789-820.

[19] R. Kruse and Y. Wu, A randomized milstein method for stochastic differential equations with non-differentiable drift coefficients, Discrete Contin. Dyn. Syst. Ser. B, 24 (8) (2019), 3475-3502.

[20] X. Li, X. Mao and G. Yin, Explicit numerical approximations for stochastic differential equations in finite and infinite horizons: truncation methods, convergence in pth moment and stability, IMA J. Numer. Anal. 39 (2) (2019), 847-892.

[21] M. Magdziarz, Stochastic representation of subdiffusion processes with time-dependent drift, Stochastic Process. Appl. 119 (10) (2009), 3238-3252. 
[22] M. Magdziarz and R. L. Schilling, Asymptotic properties of Brownian motion delayed by inverse subordinators, Proc. Amer. Math. Soc. 143 (10) (2015), 4485-4501.

[23] X. Mao, Stochastic Differential Equations and Applications, second ed., Horwood, Chichester, UK, 2007.

[24] X. Mao, The truncated Euler-Maruyama method for stochastic differential equations, J. Comput. Appl. Math. 290 (2015), 370-384.

[25] M. M. Meerschaert and H. Scheffler, Limit theorems for continuous-time random walks with infinite mean waiting times, J. Appl. Probab. 41 (3) (2004), 623-638.

[26] M. M. Meerschaert and P. Straka, Inverse stable subordinators, Math. Model. Nat. Phenom. 8 (2) (2013), 1-16.

[27] G. N. Milstein and M. V. Tretyakov, Stochastic Numerics for Mathematical Physics, Scientific Computation, Springer-Verlag, Berlin, 2004.

[28] E. Nane and Y. Ni, Stability of the solution of stochastic differential equation driven by time-changed Lévy noise, Proc. Amer. Math. Soc. 145 (7) (2017), 3085-3104.

[29] E. Platen and N. Bruti-Liberati, Numerical solution of stochastic differential equations with jumps in finance, volume 64 of Stochastic Modelling and Applied Probability, Springer-Verlag, Berlin, 2010.

[30] P. Przybylowicz and P. Morkisz, Strong approximation of solutions of stochastic differential equations with time-irregular coeffcients via randomized Euler algorithm, Appl. Numer. Math. 78 (2014), 80-94.

[31] S. Sabanis, A note on tamed Euler approximations, Electron. Comm. Probab. 18 (47) (2013), 1-10. 
[32] S. Umarov, M. Hahn and K. Kobayashi, Beyond the triangle: Brownian motion, Ito calculus, and Fokker-Planck equation-fractional generalizations. World Scientific Publishing Co. Pte. Ltd., Hackensack, NJ, 2018.

[33] X. Wang and S. Gan, The tamed Milstein method for commutative stochastic differential equations with non-globally Lipschitz continuous coefficients, J. Difference Equ. Appl. 19 (3) (2013), 466-490.

[34] Q. Wu, Stability analysis for a class of nonlinear time-changed systems, Cogent Math. 3 (2016), Art. ID 1228273, 10 pp.

[35] Z. Zhang and H. Ma, Order-preserving strong schemes for SDEs with locally Lipschitz coefficients, Appl. Numer. Math. 112 (2017), 1-16.

[36] W. Zhang, M. Song and M. Liu, Strong convergence of the partially truncated Euler-Maruyama method for a class of stochastic differential delay equations, J. Comput. Appl. Math. 355 (2018), 114-128.

[37] X. Zhang and C. Yuan, Razumikhin-type theorem on time-changed stochastic functional differential equations with Markovian switching. Open Math. 1 (17) (2019), 689-699.

[38] X. Zong, F. Wu and C. Huang, Convergence and stability of the semitamed Euler scheme for stochastic differential equations with non-Lipschitz continuous coefficients, Appl. Math. Comput. 228 (2014), 240-250. 